\title{
Una comprensión epistemológica de la psicopedagogía
}

\author{
AN EPISTEMOLOGICAL COMPREHENSION OF PSYCHOPEDAGOGY
}

Dr. Emilio Ortiz (eortiz@ict.uho.edu.cu) y Mg. María de los Ángeles Mariño (mariaa@ict.uho.edu.cu) Universidad de Holguín Oscar Lucero Moya (Holguín, Cuba)

\begin{abstract}
Psychopedagogy has experienced a great scientific development since 20th century as a result of disciplinary integration of psychology and pedagogy. However, epistemological problems of its professional and research practices have not been taken into account in scientific literature due to its theoretical complexity, insufficient attention by professionals who research in this discipline and perhaps for underrate general theoretical problems of sciences. The objective of this article is to offer different arguments in favour of a scientific status of psychopedagogy as an interdisciplinary science, according to logical and historical assessment and the same way the determination of main epistemological problems that it confronts.
\end{abstract}

Key words: psychopedagogy, epistemology, psychology, pedagogy, interdisciplinary.

\section{Resumen}

La psicopedagogía ha venido manifestando un gran desarrollo desde el siglo XX, como resultado de la integración disciplinar de la psicología y la pedagogía. Sin embargo, los problemas epistemológicos asociados a su práctica investigativa y profesional no han sido abordados en la literatura científica, debido a su complejidad teórica, a una atención insuficiente por parte de los profesionales que investigan en esta disciplina y quizás a un menosprecio por los problemas teóricos generales de las ciencias. El objetivo de este artículo es ofrecer varios argumentos a favor del estatus científico de la psicopedagogía como ciencia interdisciplinar, a partir de una valoración histórica y lógica de su evolución, así como la precisión de los problemas epistemológicos fundamentales que afronta.

Palabras clave: psicopedagogía, epistemología, psicología, pedagogía, interdisciplinariedad

\section{Introducción}

Entre las ciencias que han manifestado en la actualidad una tendencia interdisciplinar está la psicopedagogía, a partir del desarrollo previo alcanzado por la psicología y la pedagogía como ciencias independientes. Las cuestiones epistemológicas asociadas a esta creciente integración aparecen de manera incipiente en la literatura científica especializada, debido a la preocupación de los profesionales de la educación por profundizar en los contenidos relacionados con esta nueva disciplina, los cuales tienen un gran valor teórico y metodológico.

El objetivo de este artículo es ofrecer varios argumentos a favor del estatus científico de la psicopedagogía como ciencia interdisciplinar, a partir de una valoración histórica y lógica de su evolución, así como de la precisión de los problemas epistemológicos fundamentales que afronta.

Según Ursua (1981) la epistemología es útil si concierne a la ciencia como tal, si se ocupa de auténticos problemas que surgen a lo largo de la investigación científica, si propone soluciones consistentes en teorías y metodologías rigurosas e inteligibles y si es capaz de criticar programas y resultados, así como sugerir enfoques nuevos y promisorios. 
Por su parte Piaget plantea que las epistemologías contemporáneas devienen en una constante revisión de sus principios e instrumentos de conocimiento y considera que deben existir tres condiciones para tratar de modo útil el conocimiento: (a) la necesidad de conocer el empleo efectivo de los principios, (b) nociones o métodos en el terreno mismo (práctica científica) y (c) la necesidad no solo de la intuición sino de la técnica logística. No se puede prescindir de la lógica ni aun para superarla y que todo análisis epistemológico debe incluir, además de los problemas de validez formal, los problemas fácticos que incumben al papel y a las actividades del sujeto en el conocimiento. Como métodos de la epistemología contemporánea este mismo autor plantea el análisis directo, el análisis formalizante, el método genético, el método histórico crítico y el método psicogenético y la epistemología genética. Este último método es el preponderante en la concepción psicogenética del desarrollo, en el que Piaget integra aportes de la psicología y la pedagogía y que ha sido muy tenido en cuenta dentro del campo educativo.

Pero en la actualidad coexisten diferentes concepciones epistemológicas sobre el quehacer de la psicopedagogía, en dependencia de los referentes teóricos y metodológicos que las sustentan, los cuales no deben ser subestimados por su valor, como por ejemplo, la escuela de Epistemología Genética de Piaget, la escuela Histórico Cultural de Vigotsky y la corriente Humanista en la Psicología (Rogers). Dichas concepciones asumen a la psicología y la pedagogía de manera integrada, pero desde enfoques diferentes.

De acuerdo con Heitger se necesita una fundamentación filosófica de la pedagogía para evitar la inmediatez de la praxis, un empobrecimiento de la realidad y una reducción del pensamiento científico a la tecnología. El argumento de Heitger es que "una pedagogía filosófica abre la perspectiva de una praxis pedagógica que no se agota en la instrumentalización y en la innoble servidumbre a intereses sociales o políticos, sino en las consecuencias de su inmanente aspiración a una actuación vinculante" (1993:97).

Este criterio es muy importante porque está dirigido contra aquellos científicos que, desde finales del siglo $\mathrm{XX}$, ha venido promoviendo un cuestionamiento al carácter científico de la pedagogía, al considerarla una mera tecnología, ya que ella se restringe a aplicar los aportes de la psicología en el proceso formativo.

Los partidarios del carácter tecnológico de la pedagogía (Skinner, Talízina) se basan en postulados epistemológicos pragmáticos y conductistas porque resaltan solamente su carácter instrumental, pero desdeñan el valor heurístico de la teoría en la búsqueda de explicaciones rigurosas a los fenómenos, destacando solamente la posibilidad de provocar cambios objetivos y constatables en los educandos a través de una medición objetiva, como fundamento del logro de cambios sociales. Merani apunta que los problemas comunes de la psicología y la pedagogía son los relacionados con la cuestión del objeto y del sujeto, ya que entre ellos se manifiesta una dialéctica donde se integra lo psicológico con lo pedagógico.

Precisamente es en la relación objeto-sujeto donde se evidencian con nitidez los diferentes postulados epistemológicos que fundamentan a la psicopedagogía y que se reflejan en las concepciones existentes. Lo que para otras ciencias es normal al plantearse una relación sujeto-objeto de conocimiento, en las ciencias sociales en general y para la psicopedagogía en particular, el docente es tan sujeto como el alumno y esa relación contiene una esencia peculiar.

El énfasis en que el alumno es un objeto está en la base de los postulados educativos conductistas por considerar que lo subjetivo no es medible científicamente. Por su parte, la importancia del sujeto caracteriza a las posiciones humanistas porque destacan la riqueza psicológica de las personas, especialmente en su interacción con los demás como base del proceso formativo. Es así como las concepciones humanistas y vigotskianas han resaltado el valor de la subjetividad y su enriquecimiento constante como principio y fin de la educación.

\section{La psicopedagogía como ciencia interdisciplinaria aplicada}

El término psicopedagogía es relativamente reciente. Desde hace algunos años se viene reiterando dentro de las ciencias de la educación, no solo como campo del conocimiento científico, sino como carrera universitaria, y por 
tanto, como profesión en el mundo.

Los antecedentes están en la psicología aplicada a la educación. Se ubica su surgimiento a los finales del siglo XIX por el interés de los psicólogos en las características de la psiquis infantil en relación con las tareas de la enseñanza y la educación, así como por la necesidad de organizar el proceso pedagógico sobre una base psicológica, es decir, conocer al hombre antes de educarlo (Yaroschevsky).

En este análisis histórico se observa un interés y preocupación constante en hallar una fundamentación psicológica de la pedagogía. Se remonta a la historia de la cultura grecolatina, mencionando a Platón y Aristóteles en cuanto a sus referencias a la constitución de la personalidad del individuo y a la forma de cómo orientar a la naturaleza humana para conseguir mejores resultados (Prieto).

En los Estados Unidos se destaca a W. James (1842-1910) como fortalecedor de la interrelación e interdisciplinariedad de la psicología y la pedagogía, así como la obra de E. Thorndike (1874-1946) que contribuyó a la rigurosidad y autonomía de la psicología de la educación. También son mencionados el suizo E. Claparede (1873-1940), los franceses A. Binet (1857-1911) y T. Simón (1858-1918), el alemán K. Lewin (1890-1947) y el norteamericano J. Watson (1871-1958).

En Latinoamérica un numeroso abanico de fenómenos psicológicos ha tenido aplicación directa al campo de la educación, como terreno de muchos y patrimonio exclusivo de nadie. Desde el punto de vista histórico a las relaciones entre la psicología y la pedagogía se le llamó psicopedagogía por influencia europea, criterio que fue siendo desplazado por el término psicología educativa o educacional, a partir de los años 50 por influencia norteamericana. Y que los orígenes de la psicología como profesión están íntimamente relacionadas con las aplicaciones pedagógicas, un ejemplo de ello fue la creación y aplicación de los test mentales (Orantes).

Las relaciones entre pedagogía y psicología tienen en común al ser humano, la segunda para estudiarlo y la primera para formarlo, pero marcharon por sendas paralelas. El movimiento de la Escuela Nueva, cuyos precursores fueron Rousseau (1712-1778), Pestallozi (1746-1827), Froebel (1782-1849) y Herbart (1782-1852), estimuló la integración entre ambas porque destacó la necesidad de apoyarse en las características psicológicas de los alumnos para poder educarlos de acuerdo con las exigencias de la época. En la actualidad la psicopedagogía debe preocuparse por el hombre concreto, conocerlo y educarlo, no se puede formar a quien no se conoce.

El hecho de que exista una precedencia histórica para la integración de las ciencias psicológicas y pedagógicas, no quiere decir que se logre ineluctablemente porque las condiciones histórico-sociales tienen su contribución en el desarrollo científico. El origen y evolución de la psicología como disciplina independiente está fuertemente impregnada de improntas pedagógicas dondequiera que se fue arraigando esta ciencia en el mundo, pero el marco socio-histórico jugó siempre un papel decisivo.

Se parte del supuesto que en el desarrollo científico ocurren dos tendencias al unísono: el crecimiento de una multiplicidad de teorías y la formación de una teoría general unificada, ambas constituyen dos etapas en el desarrollo del conocimiento que se presuponen mutuamente.

A medida que la psicología y la pedagogía fueron enriqueciendo sus respectivos cuerpos teóricos, se iban gestando las condiciones para la conformación de una teoría integrada de ambas. Es decir, que en la psicología de la educación se están produciendo procesos analítico-sintéticos, o mejor dicho, de abstracción-generalización mutuamente condicionados. El primer proceso corresponde al crecimiento teórico particular y el segundo a la unificación de ellas.

Como resultado del desarrollo del saber científico aparecen ciencias intermedias o de transición que no son el resultado formal de unas utilizando métodos de otras, sino debido a la profundización del conocimiento, el cual conduce al descubrimiento de regularidades complejas, de niveles de desarrollo superiores, de una mayor revelación de la interconexión universal de los fenómenos de la naturaleza y de la sociedad (Kedrov). En la historia de las ciencias 
ha ocurrido reiteradamente que una nueva disciplina surge de las relaciones entre las disciplinas existentes (Betchel y Abrahamson). Ambos autores, en diferentes épocas y contextos, se están refiriendo al mismo hecho del surgimiento de nuevas fronteras interdisciplinarias, primero en las ciencias naturales, como por ejemplo, la bioquímica y, posteriormente, en las ciencias sociales, como es el caso de la psicopedagogía.

El conocimiento psicopedagógico es obvio que no puede quedar agotado con los aportes de la psicología o la pedagogía solamente, por el contrario, se nutre de todas las ciencias que estudian a los seres humanos y a la sociedad de una forma directa e indirecta, lo que enriquece el nivel teórico general de las conceptualizaciones de esta ciencia y compulsan a la obligatoria pedagogización de las ciencias, debido a las urgencias por delimitar y precisar la salida educativa de los resultados tan aparentemente lejanos de la pedagogía, como son la inteligencia artificial y las tecnologías de la información y la comunicación, entre otras. Y a la vez condiciona la aparición de nuevos y más complejos problemas epistemológicos.

De manera que la psicopedagogía posee antecedentes históricos y lógicos que le permiten adquirir un status científico como ciencia o disciplina intermedia con las demás ciencias psicológicas, como por ejemplo, con la psicología general, la psicología de la personalidad, la psicología del aprendizaje, la psicología del desarrollo y la psicología de la comunicación. Pero a la vez se integra también a las ciencias de la educación por su esencia interdisciplinar.

La psicopedagogía es una ciencia aplicada que no solo obtiene conocimientos teóricos sino que los emplea en función del proceso educativo, dentro de los cuales están la subjetividad de los alumnos y de los profesores, así como las interacciones que establecen ambos dentro de un contexto sociocultural e histórico determinado.

Por tanto, es considerada ante todo una disciplina científica aplicada por el carácter concreto y particular de su objeto: el proceso educativo, con un núcleo teórico conceptual bien delimitado e integrado por diferentes teorías, principios, categorías y modelos que permiten describir, fundamentar y explicar los fenómenos y procesos que ocurren dentro de dicho objeto, así como diferentes métodos y procedimientos dirigidos a conocer e intervenir para perfeccionar este proceso.

La aceptación del proceso educativo como su objeto esencial presupone concebirlo en un sentido general e integral porque dentro de él existe una amplia gama de fenómenos de enseñanza y aprendizaje incluidos, los cuales pueden ser objeto de estudio de manera independiente y analítica, pero sin apartarlos del contexto amplio que es educativo, dirigido a la formación, el desarrollo y el perfeccionamiento de los educandos.

En la labor cotidiana del profesional de la educación, la psicopedagogía no es la única ciencia útil, pero juega un papel fundamental porque la subjetividad de los actores claves constituye un elemento esencial, que al ser obviado por ignorancia o menosprecio puede impedir cualquier intento innovador y de perfeccionamiento del proceso pedagógico en las complejas condiciones en que se desarrolla la educación del siglo XXI.

Pero en el desarrollo del saber psicopedagógico intervienen también otras ciencias, por lo que este fenómeno es mucho más complejo. Autores como Vázquez, García y Martínez y Castillejo relacionan a la pedagogía no solo con la psicología, sino también con la lingüística, la antropología, la filosofía, la historia, la ecología, la economía, la estadística, la cibernética, la informática y hasta con las tecnología de la información por su impacto en la educación, razón por la cual le confieren a la pedagogía el carácter de ciencia cognitiva en su amplia concepción.

También posee un componente instrumental importante como ciencia aplicada, capaz de resolver los problemas prácticos asociados a su objeto. Por estas razones es que se afirma que la psicopedagogía es a la vez ciencia y tecnología, al emplear conocimientos científicos al integrarse y retroalimentarse mutuamente la ciencia y la tecnología. 
En Cuba, como parte del campo socialista, se difundieron los logros innegables de la pedagogía y la psicología, junto con los males que lastraban sus vínculos. Por ejemplo, en la década del 70 fue creada la carrera de psicopedagogía dentro de las universidades pedagógicas, con un plan de estudios que contenía asignaturas de ambas ciencias, pero sin lograr la necesaria unidad interna de una relación interdisciplinaria, ya que estaban separadas las materias de pedagogía y de psicología. Por ejemplo, teoría de la enseñanza, teoría de la educación, psicología general, psicología de las edades, psicopatología, psicología pedagógica, historia de la psicología e historia de la pedagogía, entre otras. A inicio de los años 90 la carrera fue cerrada en todo el país por decisiones políticas a partir de criterios errados.

En la actualidad esta carrera se reinició con el mismo nombre en todas las universidades pedagógicas del país, pero con un diseño curricular diferente que refleja mejor el carácter interdisciplinario de la psicopedagogía, al integrar contenidos en las diferentes disciplinas y asignaturas del plan de estudios, aunque todavía incompleto, de acuerdo con el desarrollo que ha tenido esta ciencia interdisciplinaria en estos momentos.

\section{Algunos problemas epistemológicos de la psicopedagogía}

Sobre el estatus científico de la psicopedagogía existen criterios divergentes entre los investigadores. Algunos defienden su existencia independiente y otros la niegan a favor las disciplinas de base. El propio proceso de unificación de las ciencias tradicionales en nuevas síntesis interdisciplinarias revela este problema ante un impulso creciente de integración de los conocimientos psicológicos y pedagógicos, que reafirma un lugar para ella en sus complejas relaciones con otras ciencias sociales y técnicas.

Existen varios argumentos teóricos a favor del estatus epistemológico de la psicopedagogía como nueva ciencia interdisciplinaria:

- La imposibilidad actual de estudiar los fenómenos educativos desde la psicología o desde la pedagogía de forma paralela. Para el logro de un estudio verdaderamente científico es imprescindible que su objeto de investigación sea de carácter psicopedagógico, de lo contrario se estaría parcelando la realidad educativa.

- La acumulación cuantitativa y cualitativa de conocimientos científicos cuya esencia es psicopedagógica, contribuye al desarrollo de un corpus teórico y metodológico que sustenta a esta disciplina.

- El aporte de métodos y técnicas investigativas que ya no son patrimonio exclusivo de la psicología o de la pedagogía, facilitan la obtención de conocimientos científicos más integrales que brindan respuestas oportunas y certeras a los problemas de la práctica educativa.

Estos argumentos permiten defender la existencia de una disciplina psicopedagógica con su objeto, con métodos y con un sistema de conocimientos abultado que no pueden ser restringidos a las ciencias de base porque no constituye una suma de ellas, sino una integración de nivel superior en lo teórico y metodológico, lo que ha estimulado la aparición y afianzamiento de la profesión de psicopedagogo, y por tanto, de su formación profesional como carrera universitaria. Sin embargo, es todavía insuficiente la investigación teórica dirigida a ofrecer mayores argumentos científicos que la fundamente como nueva disciplina, así como en su denominación, ya que algunos la conciben como psicología pedagógica o psicología de la educación, términos que no llegan a reflejar la esencia de su contenido interdisciplinario.

El empirismo predominante en las investigaciones educacionales, las pocas exigencias por elevarse por encima de los datos para elaborar generalizaciones teóricas, unido a la falta de una cultura verdaderamente científica de los profesionales de la educación, han contribuido a la inexistencia de elaboraciones teóricas generales para fundamentarla mejor y ofrecerle más coherencia epistemológica a la psicopedagogía.

La conformación de un sistema epistémico de la psicopedagogía constituye un reto urgente, pero primero hay que ganar conciencia entre los investigadores y psicopedagogos de su pertinencia y necesidad, aunque existen algunos intentos, como por ejemplo de Prieto, la cual aporta elementos valiosos, específicamente en cuanto a la psicología de la educación, en cuatro criterios de diferenciación: "el de dominio material, dominio de estudio, nivel de 
integración teórica, y el de las contingencias históricas de la Psicología de la Educación” (Prieto 1985:177), con la precisión de elementos valiosos para fundamentar el tránsito interdisciplinar desde la psicología de la educación. Por su parte, Orantes (1993) realiza un interesante análisis sobre el desarrollo de esta rama de la psicología en Latinoamérica, con criterios muy interesantes y novedosos que demuestran un desarrollo peculiar de esta ciencia en esta zona geográfica y cultural.

En un evento tan importante y abarcador como fue la convención internacional Pedagogía '95, desarrollado en La Habana, fueron muy pocos los trabajos referidos a este corte epistemológico (Valera, Álvarez, López). A los dos años siguientes, en el evento Pedagogía '97 la situación no fue muy diferente, por lo que la situación finisecular no era halagüeña. Ya en el siglo XXI, aunque se ha incrementado el número de investigaciones de corte psicopedagógico y de publicaciones que abordan este problema, se mantiene la necesidad de continuar profundizando y aportando nuevos elementos sobre su estatus científico.

La ampliación del concepto de educación constituye un aspecto importante dentro del quehacer profesional e investigativo, pues de manera progresiva el proceso pedagógico ha ido expandiéndose de los muros académicos hacia la comunidad y la familia, en contraposición con las concepciones tradicionales que restringen el concepto al marco de las instituciones escolares. Orantes (1993) se refiere a las concepciones que la conciben como el proceso amplio de la socialización humana, el cual se complementa con el enfoque comunitario de la educación prevaleciente en Latinoamérica.

La lógica que subyace en este criterio es que el proceso formativo humano adquiere un carácter complejo por recibir múltiples influencias provenientes no solo de las instituciones escolares, sino también de la familia y la comunidad. Por tanto, la educación es escolar, familiar y comunitaria y la psicopedagogía no puede desconocer este hecho en su conceptualización teórica y metodológica.

Pero el papel preponderante de la escuela no puede ni debe ser subestimado dentro de esta concepción tan abarcadora de la educación, por ser la mejor preparada para dirigir el proceso pedagógico en coordinación con las otras instituciones sociales ya mencionadas. Lejos de diluirse, la función de la escuela entre las demás, queda reforzada con más responsabilidades por su gran pertinencia social, ya que en la familia y en la comunidad coexisten influencias formativas con alto grado de espontaneidad y poca sistematización.

La existencia y el desarrollo de una sociología de la educación deviene como disciplina que entronca directamente con la psicopedagogía, debido al tratamiento científico que requiere el contexto social en la formación de los seres humanos. La dimensión sociológica de la educación no siempre ha sido abordada de manera explícita, aunque implícitamente lo social siempre está presente. Esta afirmación se evidencia en que los contextos sociales y culturales no se han tenido en cuenta en la extrapolación de resultados científicos de un país o de una región a otra sin las necesarias adecuaciones. Todos los problemas psicopedagógicos tienen raigambre social porque surgen y se desarrollan en determinados contextos históricos, sociales, culturales y geográficos y sin los aportes de la sociología de la educación es imposible analizarlos de manera integral y rigurosa. Por ejemplo, los intentos de aplicar los aportes del neoconductismo skineriano, de la epistemología genética piagetiana y de la concepción histórico cultural vigotskiano en Latinoamérica, sin una adecuación previa que contextualice dichos aportes, han tenido dificultades en su efectividad.

Otra disciplina importante para la psicopedagogía es la historia de la educación, la cual no debe restringirse a estudiar solamente el pensamiento pedagógico y sus condicionantes históricas, sino que incluya también a los sistemas y políticas educativas con sus tendencias actuales y perspectivas, con el fin de lograr una comprensión más completa y compleja del fenómeno educativo en sus dimensiones subjetiva, social e histórica.

La propia existencia de la psicopedagogía como disciplina científica ha condicionado la aparición de principios interdisciplinarios que no se corresponden con los puramente psicológicos o didácticos, ya que los trascienden por ser más abarcadores, aunque sin excluirlos. Un ejemplo de ello es la asunción del enfoque personológico como 
principio de destaca a la integridad de la personalidad de educandos y educadores como sujetos en el proceso formativo, aunque todavía está todavía insuficientemente sistematizado en la teoría y en la práctica educativa profesional, a pesar de su reiteración temática en investigaciones y publicaciones científicas. Se puede afirmar que el enfoque personológico en la educación ha renacido en la búsqueda de aprehender a los educandos en su integridad, no como un conjunto agregado de cualidades, características o rasgos. Sus antecedentes están en dos grandes precursores de la psicología humanista: C. Rogers y G. W. Allport. Por eso las nuevas posiciones teóricas de carácter psicopedagógico remarcan las diferencias entre sujeto y personalidad (González 1995, González y Mitjáns 1989, Arias 1995, Fernández 2005), ya que como ser indivisible posee una configuración psicológica peculiar (personalidad), pero con un activismo que se lo proporciona el carácter de sujeto. Es decir, el sujeto es un mediatizador activo de las influencias sociales y de su repercusión en la personalidad, lo cual permite explicar comportamientos simuladores o aparentes para no entrar en contradicción con las exigencias del proceso pedagógico, así como el carácter no lineal ni automático del valor intrínseco de las actividades educativas que se realizan.

El rescate de la personalidad en la educación conduce a la aceptación de la subjetividad humana como una realidad ontológica, a la necesidad del conocimiento científico dentro de las reflexiones epistemológicas, así como la reivindicación de lo psicológico y cualitativo de la personalidad frente al predominio del positivismo en las ciencias sociales, expresado en la sobrevaloración de lo experimental y tecnológico, como por ejemplo en algunos representantes de la psicología cognitiva (González 1993 y 2007).

Pérez propone la diferenciación de estos enfoques o modelos que revelan este problema: el racionalista con la utilización del método hipotético-deductivo, el paradigma proceso-producto, el análisis solo de fenómenos observables, susceptibles de medición, análisis matemático y el control experimental. Y el hermenéutico, como alternativa del anterior, con énfasis en el clima ecológico del aula, la interpretación y comprensión contra la cuantificación y el análisis matemático, con enfoque cualitativo y pluralidad de métodos para comprender la realidad.

El enfoque conductista en la educación, con su desmedido énfasis en los cambios externos como resultado del aprendizaje, fue conduciendo a esquematizar y a externalizar demasiado los resultados del proceso pedagógico. La individualidad humana, como punto de partida y de llegada en el proceso formativo, requiere ser destacado y ubicado en el centro de la concepción psicopedagógica y su repercusión metodológica debe traducirse en la investigación más profunda, como por ejemplo, el estudio de casos y la predicción científica a nivel personológico y no solamente a nivel conductual.

La reconsideración epistemológica de la concepción psicopedagógica en la educación presupone no solo cambios en los sistemas epistémicos de esta disciplina de los profesores, sino también de los alumnos. Daudinot plantea que dichos sistemas epistémicos van cambiando con el desarrollo social de las ciencias, lo cual es aplicable a la educación porque los profesores se basan en determinados sistemas epistémicos, es decir, antes de imponérselos a los alumnos, hay que argumentarlos y razonarlos con estos. El problema del aprendizaje por descubrimiento y la relación entre los métodos científicos y los métodos de enseñanza, constituyen cuestiones derivadas de este problema (Ciccilini y Sicca).

Toda la polémica actual sobre la activación de la enseñanza, la estimulación de la inteligencia y de la creatividad en la educación, así como la reconsideración de error como parte del proceso de aprendizaje, refleja una concepción nueva de dicho proceso, que primero debe penetrar en la conciencia de los claustros de profesores, para posteriormente llevarlo a los educandos de forma fluida y sistemática y no introducirlo de manera externa, esquemática y a regañadientes.

Estas nuevas ideas que revolucionan poco a poco los cauces tradicionales de la educación constituyen una nueva concepción del mundo educativo sin la pretensión de negar el aporte de la tradición, por el contrario, deben rescatarse e incorporarse a los nuevos enfoques las conquistas indiscutibles del pensamiento educativo porque no se contraponen, sino que se complementan y hasta se presuponen. 
La revalorización de estudio de casos y de lo cualitativo en el estudio de la personalidad repercute en la propia investigación psicopedagógica, pues han proliferado concepciones que han absolutizado lo cuantitativo en las investigaciones educativas, como por ejemplo, la representatividad del muestreo con un criterio solamente probabilístico, desconociendo las peculiaridades del objeto de estudio y de los sujetos investigados. La asunción de lo cualitativo implica tener en cuenta que los estudios científicos pueden ser intensivos sin desmerecer su rigor metodológico, es decir, no se tiene que trabajar obligatoriamente con una muestra grande de sujetos, ni el muestreo tiene que ser aleatorio.

La representatividad de la muestra debe descansar en que los sujetos seleccionados, muchas veces intencionalmente, poseen el fenómeno objeto de estudio en todas sus posibles variaciones, como representantes esas personas de la mayoría de las características poblacionales y sus modificaciones.

Frecuentemente, debido a los criterios predominantes hasta ahora, se han trabajado con grandes muestras con la tranquilidad para los investigadores que poseen la representatividad necesaria de la población, pero con el desconocimiento de si en esos grupos de sujetos están incluidas las variaciones del fenómeno analizado, lo cual puede introducir un sesgo en los datos que tergiversen por completo los resultados. Por eso es frecuente encontrar investigaciones en el campo educativo con resultados totalmente contradictorios, a pesar de haber sido ejecutadas con objetivos y métodos similares o idóneos.

La especificidad de la psicopedagogía como ciencia social provoca que existan diferencias sustanciales con la metodología de la investigación las ciencias naturales, lo cual ha penetrado junto con el enfoque positivista en las ciencias sociales en general y en la educación en particular, con grandes aparatajes experimentales y estadísticos, así como posiciones empiristas.

Como la investigación psicopedagógica tiene la sutileza de que son personas investigando a otras personas, el papel del investigador (educador) es vital en este proceso. En las ciencias naturales se produce una relación sujeto (investigador-objeto), pero en este caso es una relación sui géneris sujeto (investigador-educador) - sujeto (investigado), por lo que adquiere un matiz y una connotación diferentes el proceso indagatorio.

La intencionalidad del investigador y la relación empática que tiene obligatoriamente que establecer con los sujetos investigados, le confiere un carácter peculiar a la investigación psicopedagógica, planteando nuevos problemas a la teoría y a la metodología, pero a la vez enriqueciendo los resultados científicos mediante una relación sujeto-sujeto como toda fenomenología sociopsicológica intrínseca a los seres humanos y con muchas potencialidades por su impacto formativo.

El vínculo entre lo objetivo y lo subjetivo en los resultados científicos deviene como problema derivado de estas valoraciones sobre la investigación en psicopedagogía. El enfoque tradicional positivista plantea la búsqueda ante todo de lo objetivo como paradigma lógico en el estudio de los fenómenos naturales, pero en las investigaciones relacionadas con la formación de las personas es imposible despojarse de la comprensión del sujeto y de la subjetividad, bajo pena de perder la propia esencia de los seres humanos.

El predominio del positivismo en la educación ha determinado cierto exceso de objetivismo para limitar al máximo cualquier manifestación de la subjetividad en la obtención de los datos, cuestión contradictoria cuando se trabaja con personas que, precisamente en el proceso de formación y desarrollo de su personalidad, la evolución significativa de su mundo interno le es consustancial.

No se debe confundir la aceptación de lo subjetivo con el subjetivismo, como elementos que desvirtúa totalmente los resultados investigativos. El subjetivismo es la tergiversación y la exageración en el conocimiento de lo subjetivo, su absolutización en detrimento de la objetividad que debe poseer todo conocimiento científico. Lo objetivo y lo subjetivo son contrarios dialécticos que se niegan y se presuponen a la vez, pero no se deben contraponer como excluyentes, confundir ni identificar. Por eso en la actualidad muchos autores defienden la posibilidad y la necesidad 
de complementar la investigación cuantitativa con la cualitativa.

\section{Conclusiones}

La psicopedagogía como ciencia interdisciplinar posee estatus científico reconocido, como resultado del progresivo y constante proceso de integración de la psicología y la pedagogía, a partir de determinados antecedentes históricos en el que confluyen otras ciencias sociales muy desarrolladas en la actualidad. Por tanto, es lícito referirse a la psicopedagogía como ciencia con sus problemas, teorías y metodologías propias vinculadas con la formación y desarrollo de educandos y educadores, no solo en contextos escolarizados, sino también en la familia y en la comunidad.

A la psicopedagogía se le plantean varios problemas epistemológicos de gran vigencia y de cuya dilucidación depende el enriquecimiento de su aparato categorial, así como las nuevas propuestas de concebir la formación de las personas dentro de un entorno ecológico. Los problemas epistemológicos de la psicopedagogía y su comprensión científica se insertan totalmente dentro de los problemas sociales de las ciencias que se deben enfrentar con audacia, sabiduría y flexibilidad de pensamiento, especialmente en el campo de la educación, por constituir un terreno propicio para la reflexión teórica, así como para solucionar los urgentes retos que le presentan los procesos formativos para el logro de una educación de mayor calidad y equidad.

\section{Bibliografía}

Arias, H. 1995. La comunidad y su estudio. La Habana: Editorial Pueblo y Educación.

Fernández, L. 2005. Pensando en la personalidad. La Habana: Editorial Félix Varela.

González, F. y Mitjáns, A. 1989. La personalidad. Su educación y desarrollo. La Habana: Editorial Pueblo y Educación.

González, F. 1993. Problemas epistemológicos de la psicología. México: Universidad Autónoma de México.

González, F. 1995. Comunicación, personalidad y desarrollo. La Habana: Editorial Pueblo y Educación.

González, F. 2007. Investigación cualitativa y subjetividad: los procesos de construcción de la información. México: Mc Graw-Hill Interamericana.

Heitger, M. 1993. Sobre la necesidad de una fundamentación filosófica de la pedagogía. Revista Española de Pedagogía 194: 89-98.

Orantes, A. 1993. Panorama y perspectiva de la psicología aplicada a la educación en Latinoamérica. Revista Papeles del Psicólogo 55: 31-40.

Prieto, M. 1985. Reflexiones epistemológicas sobre la psicología de la educación. Anales de Pedagogía 3: $175-200$.

Ursua, N. 1981. Filosofía de la ciencia y metodología crítica. Bilbao: Editorial Descleé de Brower.

Recibido el 25 Ago 2013

Aceptado el 3 Dic 2013 